\title{
Predicting massive transfusion in placenta previa
}

\author{
Shigeki Matsubara $^{1} \cdot$ Hironori Takahashi $^{1} \cdot$ Yosuke Baba $^{1}$
}

Received: 2 June 2017 / Accepted: 6 June 2017 / Published online: 19 June 2017

(C) Japanese Society of Anesthesiologists 2017

Keywords Cesarean · Placenta previa · Probability · Hemorrhage $\cdot$ Transfusion

To the Editor:

We commend Kim et al. [1] for proposing a scoring system to predict massive transfusion in placenta previa (PP). We have already reported a simpler system [2]. We set the requirement of for allogeneic blood transfusion as an endpoint, which also reflects massive hemorrhage. The Kim et al. system consisted of five factors: (i) ultrasound sign of abnormally invasive placenta (AIP), (ii) previous cesarean section (CS), (iii) preterm delivery, (iv) sponge-like echo, and (v) anterior placenta. Our system consists of only three factors: (a) lacunae, (b) previous CS, and (c) placenta covering the previous CS scar. There are strong resemblances between the two: (i) represents (a), (ii) are identical to (b), and (v) is almost the same as (c). The area under the receiver operating characteristic curve was 0.84 , identical in both models.

Kim et al. employed three ultrasound signs, i.e., lacunae, loss of clear zone, and sponge-like appearance, of which we employed only the lacunae. Simplicity does not always mean superiority; however, lacunae (placental hypoechoic areas) may be easier to identify than the remaining two.

Experience may have prompted two different teams to independently devise these similar models. In daily

This comment refers to the article available at doi:10.1007/ s00540-017-2365-8.

Shigeki Matsubara

matsushi@jichi.ac.jp

1 Department of Obstetrics and Gynecology, Jichi Medical University, 3311-1 Shimotsuke, Tochigi 329-0498, Japan practice, both anesthesiologists and obstetricians need such a "probability index" rather than an "odds ratio." What factors should be incorporated in such a model should be further studied on a large population.

Author contributions S.M., H.T., and Y.B. identified the significance and wrote the manuscript.

Compliance with ethical standards

Conflict of interest We have no conflict of interest regarding this article.

Approval of Institutional Review Board Not needed.

Sources of funding None.

Patient anonymity Not applicable.

Informed consent Not applicable.

\section{References}

1. Kim JW, Lee YK, Chin JH, Kim SO, Lee MY, Won HS, Choi WJ. Development of a scoring system to predict massive postpartum transfusion in placenta previa totalis. J Anesth. 2017; doi:10.1007/s00540-017-2365-8.

2. Baba Y, Ohkuchi A, Usui R, Suzuki H, Kuwata T, Matsubara S. Calculating probability of requiring allogeneic blood transfusion using three preoperative risk factors on cesarean section for placenta previa. Arch Gynecol Obstet. 2015;291:281-5. 